\title{
Spin-orbit Interactions for Singlet-Triplet Qubits in Silicon
}

\author{
Patrick Harvey-Collard, ${ }^{1,2, *}$ N. Tobias Jacobson, ${ }^{3}$ Chloé Bureau-Oxton, ${ }^{1,2}$ Ryan M. Jock, ${ }^{2}$ Vanita Srinivasa, ${ }^{3}$ \\ Andrew M. Mounce, ${ }^{2}$ Daniel R. Ward, ${ }^{2}$ John M. Anderson, ${ }^{2}$ Ronald P. Manginell, ${ }^{2}$ Joel R. Wendt, ${ }^{2}$ Tammy Pluym, ${ }^{2}$ \\ Michael P. Lilly, ${ }^{4}$ Dwight R. Luhman, ${ }^{2}$ Michel Pioro-Ladrière, ${ }^{1,5}$ and Malcolm S. Carroll ${ }^{2, \dagger}$ \\ ${ }^{1}$ Département de physique et Institut quantique, Université de Sherbrooke, Sherbrooke (Québec) $J 1 K 2 R 1$, Canada \\ ${ }^{2}$ Sandia National Laboratories, Albuquerque, New Mexico 87185, USA \\ ${ }^{3}$ Center for Computing Research, Sandia National Laboratories, Albuquerque, New Mexico 87185, USA \\ ${ }^{4}$ Center for Integrated Nanotechnologies, Sandia National Laboratories, Albuquerque, New Mexico 87185, USA \\ ${ }^{5}$ Quantum Information Science Program, Canadian Institute for Advanced Research, Toronto (Ontario) M5G 1Z8, Canada
}

(Received 21 September 2018; revised manuscript received 25 February 2019; published 29 May 2019)

\begin{abstract}
Spin-orbit coupling is relatively weak for electrons in bulk silicon, but enhanced interactions are reported in nanostructures such as the quantum dots used for spin qubits. These interactions have been attributed to various dissimilar interface effects, including disorder or broken crystal symmetries. In this Letter, we use a double-quantum-dot qubit to probe these interactions by comparing the spins of separated singlet-triplet electron pairs. We observe both intravalley and intervalley mechanisms, each dominant for [110] and [100] magnetic field orientations, respectively, that are consistent with a broken crystal symmetry model. We also observe a third spin-flip mechanism caused by tunneling between the quantum dots. This improved understanding is important for qubit uniformity, spin control and decoherence, and two-qubit gates.
\end{abstract}

DOI: 10.1103/PhysRevLett.122.217702

Introduction.-Isotopically enriched silicon is a prime semiconductor for the implementation of spin qubits [1]. In addition to reduced spin decoherence enabled by the near absence of lattice nuclear spins [2,3], silicon is a low spinorbit coupling material for electrons that enables long spin relaxation times $[4,5]$ and low coupling to charge noise. In silicon quantum dots (QDs), recent work has shown that spin-orbit effects arise in the presence of strong electron confinement [6-11]. This enhanced interaction has been attributed to intervalley spin-orbit coupling and interface disorder $[6,7,11]$ in some works, and to broken crystal symmetries [12] at the $\mathrm{Si} / \mathrm{SiO}_{2}$ [10] or $\mathrm{Si} / \mathrm{SiGe}$ [13] interfaces in other works. Recently, Jock et al. [10] have used a singlet-triplet (ST) qubit [14,15] to probe the electron $g$-factor difference between two QDs, and found a strong magnetic-field-dependent anisotropy explained with an intravalley mechanism. This anisotropy can be exploited to enhance spin-orbit effects for spin control $[3,10]$, or suppress them for uniformity and reproducibility [16]. ST qubits are promising candidates for quantum computing, thanks to the ability to perform exchange [14], capacitive [17-19], and long-range [20-23] two-qubit gates, as well as low-frequency one-qubit operations [24].

Published by the American Physical Society under the terms of the Creative Commons Attribution 4.0 International license. Further distribution of this work must maintain attribution to the author(s) and the published article's title, journal citation, and DOI.
In GaAs devices, the use of differential dynamic nuclear polarization (DDNP) was shown to dramatically enhance the ST qubit coherence $[25,26]$, and also enable its control $[26,27]$. The DDNP technique depends on the interaction between the two-electron spin singlet $|S\rangle=(|\uparrow \downarrow\rangle-$ $|\downarrow \uparrow\rangle) / \sqrt{2}$ and spin triplet $\left|T_{-}\right\rangle=|\downarrow \downarrow\rangle$ mediated by the hyperfine coupling to lattice nuclear spins [28]. It was shown that spin-orbit interaction can couple these two states as well [29], impacting the ability to perform a DDNP by providing an alternate channel to dissipate angular momentum $[30,31]$. In light of these different works, it remains unclear what spin-orbit effects predominate in different situations, what their microscopic origins are, and how these effects will impact the operation of silicon devices.

In this Letter, we report the observation of three different spin-orbit effects in the same device using a ST qubit in isotopically enriched silicon. The first two effects are probed using $S-T_{0}$ precession and appear at different orders of perturbation theory. They consist of an intravalley $g$-factor difference effect and an intervalley spin-coupling effect. The dominant mechanism depends on the magnetic field orientation with respect to crystallographic axes. We report here a nonlinear magnetic field strength dependence, in addition to previously reported linear dependences. The third effect is probed using $S-T_{-}$spin transitions and involves a spin-flip process triggered by electron tunneling between the QDs. To measure this effect, we adapt a method previously used in GaAs [31] to our silicon system, 
where the near absence of nuclear spins otherwise prevents these transitions. We find that the enhanced spin-orbit interaction in the device strongly couples these states, as it does for GaAs devices. In fact, the spin-orbit length estimated from our measurements is only slightly smaller than bulk GaAs values, a result that is in accordance with other recent observations of strong spin-orbit effects in silicon nanodevices. This prevents us from performing DDNP of the residual ${ }^{29} \mathrm{Si}$.

The effects are modeled with an analytical microscopic intravalley theory based on broken crystal symmetries introduced in Jock et al. [10] and extended in this Letter to describe the additional intervalley effect reported here. The model involves the electron momentum only at the $\mathrm{Si}-\mathrm{SiO}_{2}$ interface, resulting in stronger-than-bulk first-order effects in the electron momentum and clear predictions that could help elucidate the microscopic origin of the enhanced spin-orbit effects in the future [32]. This Letter, as a consequence of its comprehensive view of spin orbit interactions, will affect how pulses are shaped around the uncovered transitions in silicon qubits as well as providing more detailed guidance about the implications of how samples are mounted in dilution refrigerators with respect to magnetic fields.

Methods.-The experiments are performed in a dilution refrigerator with an electronic temperature of around $300 \mathrm{mK}$. The gated silicon QD device is shown in Fig. 1. The silicon is isotopically enriched ${ }^{28} \mathrm{Si}$, with a measured $685 \mathrm{ppm}$ of residual ${ }^{29} \mathrm{Si}$. Fabrication and device crystallographic orientation are as in Jock et al. [10]; the device is from the same fabrication run but a different die and measured in a different system. Two QDs are formed,
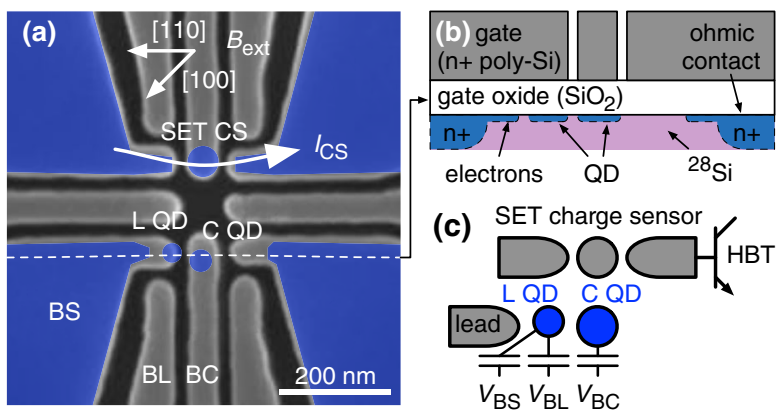

FIG. 1. (a) Scanning electron microscope image of the gate structure of the silicon QD device. The blue overlay indicates the estimated locations of electron accumulation. The crystallographic and external magnetic field $\left(B_{\text {ext }}\right)$ orientations are indicated. All experiments are performed with the magnetic field along the [100] orientation, except when otherwise specified. A SET CS is used for readout and sensing. Its current $I_{\mathrm{CS}}$ is amplified using a SiGe HBT. (b) Schematic lateral view of the device structure (along the dashed line). (c) Conceptual view of the two QDs, the single-lead reservoir, the CS and the predominant QD-gate capacitive couplings. The $\mathrm{BL}$ and $\mathrm{BC}$ gates are used for fast electrical control of the $\mathrm{L}$ and $\mathrm{C}$ QDs, respectively. one under the bottom source (BS) gate and one under the bottom center $(\mathrm{BC})$ gate. The bottom left $(\mathrm{BL})$ and $\mathrm{BC}$ gates are used for fast control of the left (L) and center (C) QD charge occupations $\left(N_{\mathrm{L}}, N_{\mathrm{C}}\right)$ and interdot detuning $\epsilon$.

The double QD is biased in a two-electron charge configuration to form a ST qubit. The L QD has a ST splitting of $243 \mu \mathrm{eV}$ and the C QD has one of $185 \mu \mathrm{eV}$ [the latter measured in a $(3,2)$ configuration to avoid charge latching]. Spin readout is performed with a direct enhanced latching readout, as described in Harvey-Collard et al. [33], and using a single-electron transistor (SET) in series with a SiGe heterojunction bipolar transistor (HBT) cryoamplifier [34]. Triplet return probabilities $P(T)$ are calculated from the average of readout traces referenced to a known charge configuration to eliminate the slow charge sensor (CS) current fluctuations.

The external magnetic field $B_{\text {ext }}$ is applied in-plane along the [100] or [110] crystallographic orientations. The [100] orientation is used for all the experiments unless otherwise specified. The [110] orientation was obtained by rotating the sample in a separate cooldown. The device parameters (voltages, ST splittings, etc.) remained very similar between cooldowns, except for slight changes in the tunnel couplings.

Results. - A charge stability diagram of the two-electron double QD and the typical location of the pulse sequence steps are shown in Fig. 2. We use rotations between the $|S\rangle$ and $\left|T_{0}\right\rangle=(|\uparrow \downarrow\rangle+|\downarrow \uparrow\rangle) / \sqrt{2}$ states to measure the difference in Zeeman energy perpendicular to the quantization axis $\Delta E_{z}$ between the two QDs. These rotations appear with the application of an external magnetic field $B_{\text {ext }}$, as reported in Jock et al. [10], in spite of the (relative) absence of lattice nuclear spins or magnetic materials. The inhomogeneous dephasing time saturates at $T_{2}^{\star}=3.4 \pm 0.3 \mu \mathrm{s}$ after $2 \mathrm{~h}$ of data averaging. This value is consistent with magnetic noise from residual ${ }^{29} \mathrm{Si}$ hyperfine coupling with the electron spins, and with other reported values $[2,8,10,35,36]$.

To investigate the physical origin of the $S-T_{0}$ rotations, we vary the strength of $B_{\text {ext }}$ along two orientations measured in successive cooldowns. We identify different dominant spin-orbit mechanisms for these two orientations, with the results summarized in Fig. 3.

The first mechanism is a first-order intravalley effect observed both in this device and in Jock et al. [10]. The Zeeman drive is a difference in effective Landé $g$ factor $\Delta g$ between the two QDs:

$$
\Delta E_{z}=\Delta g \mu_{\mathrm{B}} B_{\mathrm{ext}} .
$$

This effect dominates in the [110] field orientation. It is not predicted to depend on the double QD orientation, as shown by the different positions for the two QDs in this Letter compared with Jock et al. [10]. 


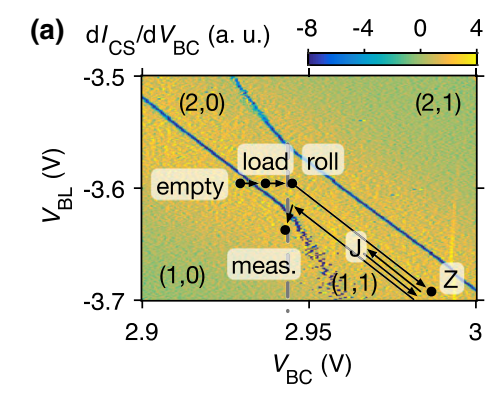

(b)

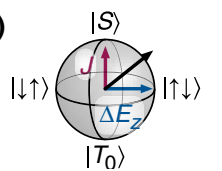

(c)

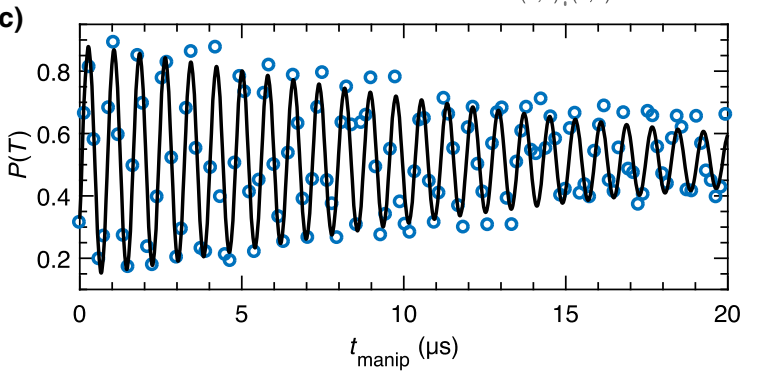

FIG. 2. (a) Charge stability diagram of the two-electron double QD. The typical pulse sequence steps are indicated. They consist of an emptying step (empty) where the charge occupancy is reset and the current referenced, a load step (load) for singlet or mixture preparation, a transient point (roll) to set the pulse trajectories and rates, some steps in $(1,1)$ (points $\mathrm{Z}$ and $\mathrm{J}$ ) for spin control, a return to "roll" step, and an enhanced latching readout step (meas.). The roll point can be placed on either sides of the $S-T_{-}$anticrossing depending on the goals. More details about the pulses can be found in the Supplemental Material Secs S1 and $\mathrm{S} 2$ [41]. (b) ST qubit Bloch sphere and pulse sequence for $S-T_{0}$ rotations. (c) Rotations between the $|S\rangle$ and $\left|T_{0}\right\rangle$ states vs the manipulation time $t_{\text {manip }}$. Those are enabled by a large $B_{\text {ext }}=1 \mathrm{~T}$ in the [100] direction, in spite of the relative absence of nuclear spins or magnetic materials. Optimal visibility is achieved in the rapid adiabatic passage (RAP) regime [24,37] (see the Supplemental Material Sec. S1 [41]). The visibility is 73\%, limited largely by preparation and readout errors. The dephasing time is $15 \mu \mathrm{s}$ for this single trace acquired in 4 minutes.

The second mechanism, newly reported here, is consistent with an intervalley spin-orbit interaction $[6,11,38]$. The smaller and nonlinear behavior vs magnetic field from Fig. 3(b) suggests a second-order interaction with an excited valley $\left|T_{-}^{*}\right\rangle$ state, as shown in Fig. 3(c). For simplicity, we consider only the QD with the lowest valley splitting $E_{\mathrm{VS}}$. In the other QD, this interaction is suppressed by the larger $E_{\mathrm{Vs}}$. Using perturbation theory, we have

$$
\begin{aligned}
E_{S}-E_{T_{0}}= & \left(E_{S}^{(0)}-E_{T_{0}}^{(0)}\right) \\
& +\left(\frac{\left|\left\langle T_{-}^{*(0)}\left|H_{\mathrm{SO}}\right| S^{(0)}\right\rangle\right|^{2}}{E_{S}^{(0)}-E_{T_{-}^{*}}^{(0)}}-\frac{\left|\left\langle T_{-}^{*(0)}\left|H_{\mathrm{SO}}\right| T_{0}{ }^{(0)}\right\rangle\right|^{2}}{E_{T_{0}}^{(0)}-E_{T_{-}^{(0)}}^{(0)}}\right) .
\end{aligned}
$$

Here, $E_{\psi}^{(i)}$ is the energy of the state $|\psi\rangle$ at the $i$ th order, and $H_{\mathrm{SO}}$ is the spin-orbit interaction Hamiltonian. We note that

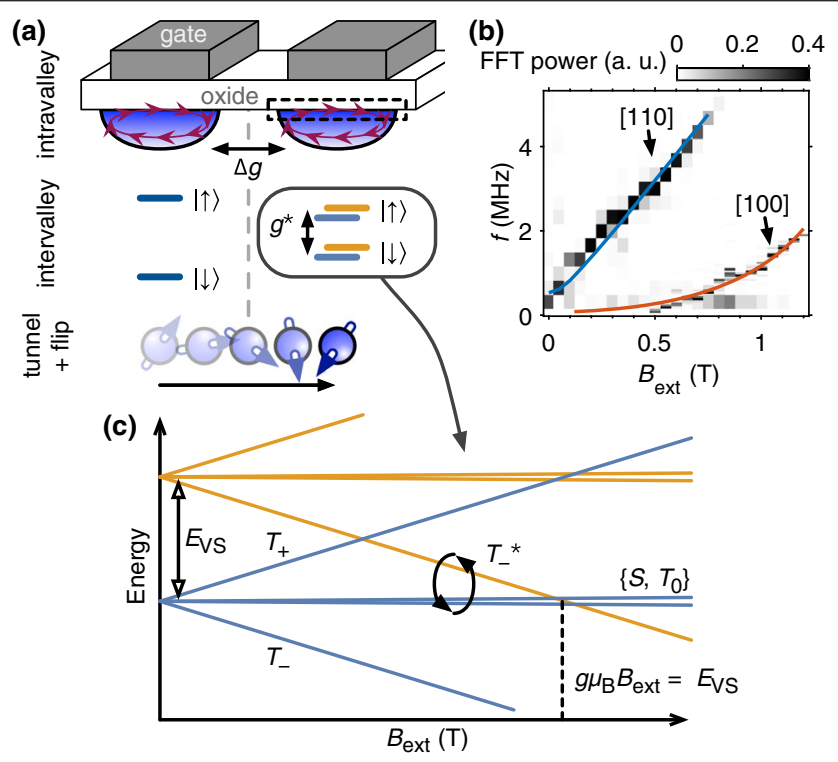

FIG. 3. (a) The three different spin-orbit effects for electrons in a silicon MOS nanodevice identified in this Letter. First, spinorbit interaction in each dot leads to a renormalization of their effective $g$ factors. This entails an interaction of the form $\Delta E_{z}=$ $\Delta g \mu_{\mathrm{B}} B_{\text {ext }}$ [see Fig. 2(b)]. Second, intervalley spin coupling can change the Landé factor $g^{*}$ of one dot in particular, leading to an interaction of the form $\Delta E_{z} \propto B_{\text {ext }}^{2} /\left(E_{\mathrm{VS}}-g \mu_{\mathrm{B}} B_{\mathrm{ext}}\right)$. Third, electron motion during a tunneling event can induce a spin flip that couples the $(2,0) S$ and $(1,1) T_{-}$states, as shown also in Fig. 4. (b) Fast Fourier transform (FFT) power of the $S-T_{0}$ rotations vs $B_{\text {ext }}$. For the [110] field orientation, the linear interdot effect dominates. For the [100] orientation, the linear interdot effect is suppressed; however, a second-order effect consistent with an intervalley mechanism is observed. The solid lines are fit to a complete Hamiltonian model detailed in the Supplemental Material Sec. S6 [41] and agree well with the simple analytical forms described above. (c) The intervalley spin coupling in one of the dots perturbs the $S-T_{0}$ energy difference at the second order through the excited valley $\left|T_{-}^{*}\right\rangle$ state. This simple model neglects the excited valley state of the other dot, which is higher in energy.

the first term on the right-hand side is the effect of Eq. (1). This first term is largely suppressed for the [100] field orientation, as in Jock et al. [10]. The second term on the right-hand side can be simplified as follows. The matrix elements $\left\langle T_{-}^{*(0)}\left|H_{\mathrm{SO}}\right| S^{(0)}\right\rangle$ and $\left\langle T_{-}^{*(0)}\left|H_{\mathrm{SO}}\right| T_{0}^{(0)}\right\rangle$ are both proportional to $B_{\text {ext }}$, as explained in the Supplemental Material Sec. S6 [41]. Therefore, Eq. (2) simplifies to

$$
\Delta E_{z}=\frac{\bar{\beta}_{C}^{2} B_{\mathrm{ext}}^{2}}{2\left(E_{\mathrm{VS}}-g \mu_{\mathrm{B}} B_{\mathrm{ext}}\right)} .
$$

Here, $\bar{\beta}_{C}$ is a measure of the Dresselhaus spin coupling of the C QD. The above treatment is simplistic but provides intuition about the physical mechanism and agrees well with the more detailed analysis of the Supplemental Material Sec. S6 [41]. We extract a value of $\bar{\beta}_{C}=0.7 \mu \mathrm{eV} \mathrm{T}^{-1}$ for the experimental data in Fig. 3(b). 


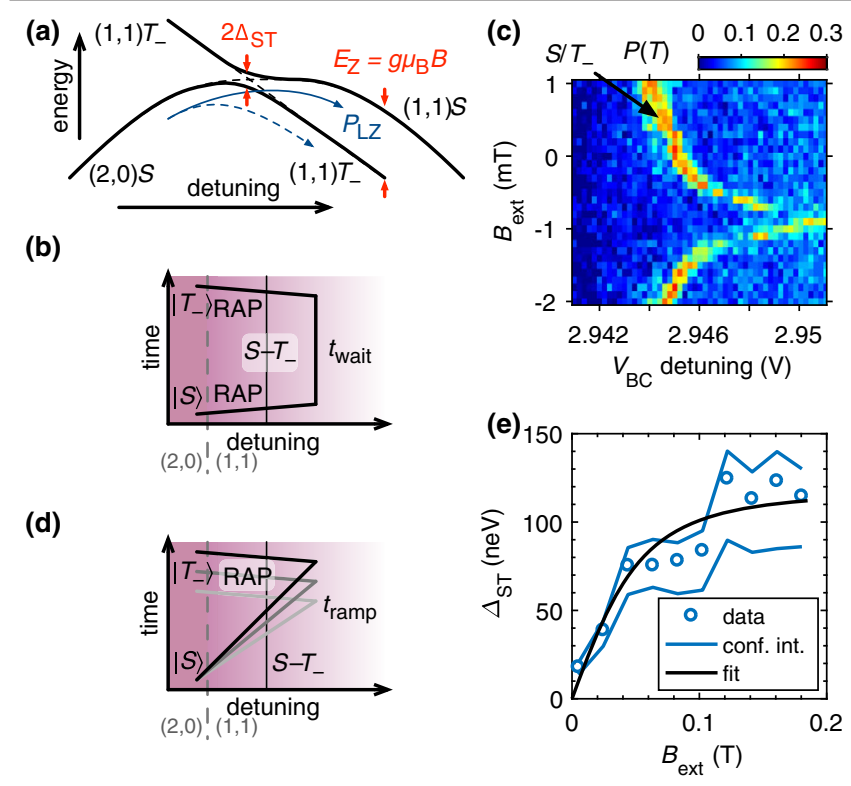

FIG. 4. (a) Anticrossing of the $|S\rangle$ and $\left|T_{-}\right\rangle$states. The separation $E_{\mathrm{Z}}=g \mu_{\mathrm{B}} B$ between the $(1,1) S$ and $(1,1) T_{-}$states is tuned with the magnetic field $B$. The coupling strength $\Delta_{\mathrm{ST}}$ can come from both a tunneling plus spin-flip mechanism that couples $(1,1)$ and $(2,0)$ states, and from the hyperfine interaction with lattice nuclear spins which couples $(1,1)$ states together. For our residual ${ }^{29} \mathrm{Si}$ concentration, the latter should be less than $0.6 \mathrm{neV}[2,35]$. (b) Pulse sequence for the spin funnel measurement. When the detuning pulse falls on the transition, mixing between $|S\rangle$ and $\left|T_{-}\right\rangle$occurs. (c) Spin funnel with $t_{\text {wait }}=20 \mu \mathrm{s}$. This measurement allows us to calibrate the tunnel coupling $t_{\mathrm{c}}$ and energy ramp rate $d E / d t$ of LZ sweeps. (d) Pulse sequence to probe the $S-T_{-}$gap through LZ transition probabilities. (e) The magnetic field dependence of the gap is fit to a simple model that includes a constant spin-orbit term $\Omega_{\mathrm{SO}}$ and charge hybridization, see the Supplemental Material Eq. (S2) [41]. The confidence interval is explained in the Supplemental Material Sec. S3 [41]. The field is in the [100] direction.

While this value is in qualitative agreement with previously inferred values for single spins $[6,11,38]$, the experimental agreement with the model in Fig. 3(b) isn't perfect. As demonstrated in Fig. 6(c) of Harvey-Collard et al. [37], we have also observed a detuning dependence of the rotation frequency (and hence $\bar{\beta}_{C}$ ). This suggests that $\bar{\beta}_{C}$ depends on the detuning via the microscopic details of the electron confinement and/or the electric field.

The ST qubit allows us to probe a third spin-orbit effect that involves a tunneling plus spin-flip mechanism, newly reported here for a silicon device. We apply the method featured in Nichol et al. [31] to measure the $S-T_{-}$gap $\Delta_{\mathrm{ST}}$. This method consists of mapping the position of the $S-T_{-}$anticrossing using the spin funnel technique [15] and probing the gap size using Landau-Zener-StückelbergMajorana (LZ) transitions [39]. The pulse sequence and results are shown in Fig. 4. The data analysis is explained in the Supplemental Material Sec. S3 [41]. We find
$\Delta_{\mathrm{ST}}=113 \pm 22 \mathrm{neV}$. This gap is expected to depend upon the orientation of both the magnetic field as well as the axis of the double QD (through the electron motion). From this value, we can estimate a spin-orbit length $\lambda_{\mathrm{SO}} \approx 1 \mu \mathrm{m}$, which is slightly smaller than the bulk value for GaAs and 20 times smaller than the bulk Si value [40]. Therefore, the spin-orbit interaction in this silicon nanoscale device is comparable to the bulk value observed in larger spin-orbit materials.

Finally, we report in the Supplemental Material Sec. S4 [41] measurements of the ST qubit relaxation time, and discuss its potential relation to the spin-orbit effects discussed here. We also explore in Supplemental Material Sec. S5 [41] the possibility to use a DDNP sequence to enhance the coherence of the qubit and induce hyperfine-driven rotations despite the isotopic enrichment [41].

Conclusion.-In summary, we report three different spin-orbit effects for electrons in an isotopically enriched silicon double QD device. We observe both coherent $S-T_{0}$ rotations and incoherent $S-T_{-}$mixing that are consistent with a spin-orbit interaction much larger than bulk silicon values. We extend an analytical theory based on broken crystal symmetries at the silicon-dielectric interface that captures first- and second-order effects. Based on this theory and the results by Jock et al. [10], we predict that the two $S-T_{0}$ effects could be eliminated with an out-of-plane magnetic field orientation since the dot-localized electron momentum at the interface vanishes in total. The $S-T_{-}$effect could potentially persist in such an orientation due to the interdot electron tunneling. Our results have implications for a variety of spin qubit encodings, like the $S-T_{0}$, the $S-T_{-}$[45] and the spin$1 / 2$ qubits, for extending the coherence of ST silicon qubits through a DDNP, for single-spin control and relaxation, and for two-qubit coupling schemes based on the exchange interaction. For example, exchange-based two-qubit gates are in many ways operations similar to those in a ST qubit [3,46-48]. Beyond qubits, our results help understand additional spin-orbit effects that emerge in nanostructures.

The authors thank David S. Simons and Joshua M. Pomeroy from National Institute of Standards and Technology for assistance with secondary ion mass spectrometry measurements on the ${ }^{28} \mathrm{Si}$ epitaxial layer. This work was performed, in part, at the Center for Integrated Nanotechnologies, an Office of Science User Facility operated for the U.S. Department of Energy (DOE) Office of Science. Sandia National Laboratories is a multimission laboratory managed and operated by National Technology and Engineering Solutions of Sandia, LLC, a wholly owned subsidiary of Honeywell International, Inc., for the DOE's National Nuclear Security Administration under Contract No. DE-NA0003525. This Letter describes objective technical results and analysis. Any subjective views or opinions that might be expressed 
in the Letter do not necessarily represent the views of the U.S. DOE or the United States Government. P. H.-C., R. M. J., and M. S.C. designed the experiments. P. H.-C. performed the experiments and analyzed the results. N. T. J. developed the theory with help from V.S. and A. M. M. Furthermore, C. B.-O. performed experiments in the [110] field orientation. P. H.-C., N. T. J., C. B.-O., R. M. J., V. S., A. M. M., and M. S.C. discussed the results. R. M. J. performed experiments on another device that establishes the reproducibility of some results. D.R.W., J.M.A., R. P. M., J.R. W., T.P., and M. S. C. designed the process flow and fabricated the devices. M. L. provided the experimental setup for the work. M. P.-L. and D. R. L. helped develop the project and provided counsel. M. S. C. supervised the combined effort, including coordinating fabrication and identifying modeling needs. P. H.-C., N. T. J., and M. S. C. wrote the manuscript with input from all coauthors.

Note added.-The authors recently became aware of simultaneous work by Tanttu et al. [49] that covers related topics.

*P.Collard@USherbrooke.ca

†mscarro@sandia.gov

[1] D. Loss and D. P. DiVincenzo, Quantum computation with quantum dots, Phys. Rev. A 57, 120 (1998).

[2] W. M. Witzel, M. S. Carroll, A. Morello, L. Cywiński, and S. Das Sarma, Electron Spin Decoherence in IsotopeEnriched Silicon, Phys. Rev. Lett. 105, 187602 (2010).

[3] M. Veldhorst, J. C. C. Hwang, C. H. Yang, A. W. Leenstra, B. de Ronde, J. P. Dehollain, J. T. Muhonen, F. E. Hudson, K. M. Itoh, A. Morello, and A. S. Dzurak, An addressable quantum dot qubit with fault-tolerant control-fidelity, Nat. Nanotechnol. 9, 981 (2014).

[4] F. A. Zwanenburg, A. S. Dzurak, A. Morello, M. Y. Simmons, L. C. L. Hollenberg, G. Klimeck, S. Rogge, S. N. Coppersmith, and M. A. Eriksson, Silicon quantum electronics, Rev. Mod. Phys. 85, 961 (2013).

[5] T. F. Watson, B. Weber, Y.-L. Hsueh, L. L. C. Hollenberg, R. Rahman, and M. Y. Simmons, Atomically engineered electron spin lifetimes of $30 \mathrm{~s}$ in silicon, Sci. Adv. 3, e1602811 (2017).

[6] C. H. Yang, A. Rossi, R. Ruskov, N. S. Lai, F. A. Mohiyaddin, S. Lee, C. Tahan, G. Klimeck, A. Morello, and A. S. Dzurak, Spin-valley lifetimes in a silicon quantum dot with tunable valley splitting, Nat. Commun. 4, 2069 (2013).

[7] M. Veldhorst, R. Ruskov, C. H. Yang, J. C. C. Hwang, F. E. Hudson, M. E. Flatté, C. Tahan, K. M. Itoh, A. Morello, and A. S. Dzurak, Spin-orbit coupling and operation of multivalley spin qubits, Phys. Rev. B 92, 201401(R) (2015).

[8] K. Eng, T. D. Ladd, A. Smith, M. G. Borselli, A. A. Kiselev, B. H. Fong, K. S. Holabird, T. M. Hazard, B. Huang, P. W. Deelman, I. Milosavljevic, A. E. Schmitz, R. S. Ross, M.F. Gyure, and A.T. Hunter, Isotopically enhanced triple-quantum-dot qubit, Sci. Adv. 1, e1500214 (2015).
[9] R. Ferdous, E. Kawakami, P. Scarlino, M. Nowak, D. R. Ward, D. E. Savage, M. G. Lagally, S. N. Coppersmith, M. Friesen, M. A. Eriksson, L. M. K. Vandersypen, and R. Rahman, Valley dependent anisotropic spin splitting in silicon quantum dots, npj Quantum Inf. 4, 26 (2018).

[10] R. M. Jock, N. T. Jacobson, P. Harvey-Collard, A. M. Mounce, V. Srinivasa, D. R. Ward, J. Anderson, R. Manginell, J. R. Wendt, M. Rudolph, T. Pluym, J. K. Gamble, A. D. Baczewski, W. M. Witzel, and M. S. Carroll, A silicon metaloxide-semiconductor electron spin-orbit qubit, Nat. Commun. 9, 1768 (2018).

[11] A. Corna, L. Bourdet, R. Maurand, A. Crippa, D. KotekarPatil, H. Bohuslavskyi, R. Laviéville, L. Hutin, S. Barraud, X. Jehl, M. Vinet, S. De Franceschi, Y.-M. Niquet, and M. Sanquer, Electrically driven electron spin resonance mediated by spin-valley-orbit coupling in a silicon quantum dot, npj Quantum Inf. 4, 6 (2018).

[12] U. Rössler and J. Kainz, Microscopic interface asymmetry and spin-splitting of electron subbands in semiconductor quantum structures, Solid State Commun. 121, 313 (2002).

[13] M. Prada, G. Klimeck, and R. Joynt, Spin-orbit splittings in $\mathrm{Si} / \mathrm{SiGe}$ quantum wells: from ideal $\mathrm{Si}$ membranes to realistic heterostructures, New J. Phys. 13, 013009 (2011).

[14] J. Levy, Universal Quantum Computation with Spin-1/2 Pairs and Heisenberg Exchange, Phys. Rev. Lett. 89, 147902 (2002).

[15] J. R. Petta, A. C. Johnson, J. M. Taylor, E. A. Laird, A. Yacoby, M. D. Lukin, C. M. Marcus, M. P. Hanson, and A. C. Gossard, Coherent manipulation of coupled electron spins in semiconductor quantum dots, Science 309, 2180 (2005).

[16] R. Li, L. Petit, D. P. Franke, J. P. Dehollain, J. Helsen, M. Steudtner, N. K. Thomas, Z. R. Yoscovits, K. J. Singh, S. Wehner, L. M. K. Vandersypen, J. S. Clarke, and M. Veldhorst, A crossbar network for silicon quantum dot qubits, Sci. Adv. 4, eaar3960 (2018).

[17] M. D. Shulman, O. E. Dial, S. P. Harvey, H. Bluhm, V. Umansky, and A. Yacoby, Demonstration of entanglement of electrostatically coupled singlet-triplet qubits, Science 336, 202 (2012).

[18] V. Srinivasa and J. M. Taylor, Capacitively coupled singlettriplet qubits in the double charge resonant regime, Phys. Rev. B 92, 235301 (2015).

[19] J. M. Nichol, L. A. Orona, S. P. Harvey, S. Fallahi, G. C. Gardner, M. J. Manfra, and A. Yacoby, High-fidelity entangling gate for double-quantum-dot spin qubits, npj Quantum Inf. 3, 3 (2017).

[20] L. Trifunovic, O. Dial, M. Trif, J. R. Wootton, R. Abebe, A. Yacoby, and D. Loss, Long-Distance Spin-Spin Coupling Via Floating Gates, Phys. Rev. X 2, 011006 (2012).

[21] S. Mehl, H. Bluhm, and D. P. DiVincenzo, Two-qubit couplings of singlet-triplet qubits mediated by one quantum state, Phys. Rev. B 90, 045404 (2014).

[22] V. Srinivasa, H. Xu, and J. M. Taylor, Tunable Spin-Qubit Coupling Mediated by a Multielectron Quantum Dot, Phys. Rev. Lett. 114, 226803 (2015).

[23] F. K. Malinowski, F. Martins, T. B. Smith, S. D. Bartlett, A. C. Doherty, P. D. Nissen, S. Fallahi, G. C. Gardner, M. J. Manfra, C. M. Marcus, and F. Kuemmeth, Spin of a 
Multielectron Quantum Dot and its Interaction with a Neighboring Electron, Phys. Rev. X 8, 011045 (2018).

[24] J. M. Taylor, J. R. Petta, A. C. Johnson, A. Yacoby, C. M. Marcus, and M.D. Lukin, Relaxation, dephasing, and quantum control of electron spins in double quantum dots, Phys. Rev. B 76, 035315 (2007).

[25] H. Bluhm, S. Foletti, D. Mahalu, V. Umansky, and A. Yacoby, Enhancing the Coherence of a Spin Qubit by Operating it as a Feedback Loop that Controls its Nuclear Spin Bath, Phys. Rev. Lett. 105, 216803 (2010).

[26] M. D. Shulman, S. P. Harvey, J. M. Nichol, S. D. Bartlett, A. C. Doherty, V. Umansky, and A. Yacoby, Suppressing qubit dephasing using real-time Hamiltonian estimation, Nat. Commun. 5, 5156 (2014).

[27] S. Foletti, H. Bluhm, D. Mahalu, V. Umansky, and A. Yacoby, Universal quantum control of two-electron spin quantum bits using dynamic nuclear polarization, Nat. Phys. 5, 903 (2009).

[28] M. Gullans, J. J. Krich, J. M. Taylor, H. Bluhm, B. I. Halperin, C. M. Marcus, M. Stopa, A. Yacoby, and M. D. Lukin, Dynamic Nuclear Polarization in Double Quantum Dots, Phys. Rev. Lett. 104, 226807 (2010).

[29] D. Stepanenko, M. Rudner, B. I. Halperin, and D. Loss, Singlet-triplet splitting in double quantum dots due to spinorbit and hyperfine interactions, Phys. Rev. B 85, 075416 (2012).

[30] M. J. Rančić and G. Burkard, Interplay of spin-orbit and hyperfine interactions in dynamical nuclear polarization in semiconductor quantum dots, Phys. Rev. B 90, 245305 (2014).

[31] J. M. Nichol, S. P. Harvey, M. D. Shulman, A. Pal, V. Umansky, E. I. Rashba, B. I. Halperin, and A. Yacoby, Quenching of dynamic nuclear polarization by spin-orbit coupling in GaAs quantum dots, Nat. Commun. 6, 7682 (2015).

[32] R. Ruskov, M. Veldhorst, A. S. Dzurak, and C. Tahan, Electron $g$-factor of valley states in realistic silicon quantum dots, Phys. Rev. B 98, 245424 (2018).

[33] P. Harvey-Collard, B. D'Anjou, M. Rudolph, N. T. Jacobson, J. Dominguez, G. A. Ten Eyck, J. R. Wendt, T. Pluym, M. P. Lilly, W. A. Coish, M. Pioro-Ladrière, and M. S. Carroll, High-Fidelity Single-Shot Readout for a Spin Qubit via an Enhanced Latching Mechanism, Phys. Rev. X 8, 021046 (2018).

[34] M. J. Curry, T. D. England, N. C. Bishop, G. Ten-Eyck, J. R. Wendt, T. Pluym, M. P. Lilly, S. M. Carr, and M. S. Carroll, Cryogenic preamplification of a single-electrontransistor using a silicon-germanium heterojunctionbipolar-transistor, Appl. Phys. Lett. 106, 203505 (2015).

[35] L. V. C. Assali, H. M. Petrilli, R. B. Capaz, B. Koiller, X. $\mathrm{Hu}$, and S. Das Sarma, Hyperfine interactions in silicon quantum dots, Phys. Rev. B 83, 165301 (2011).

[36] M. Rudolph, P. Harvey-Collard, R. Jock, T. Jacobson, J. Wendt, T. Pluym, J. Domínguez, G. Ten-Eyck, R. Manginell, M. P. Lilly, and M. S. Carroll, Coupling MOS quantum dot and phosphorous donor qubit systems, in 2016
IEEE International Electron Devices Meeting (IEDM) (IEEE, New York, 2016), pp. 34.1.1-34.1.4, https:// ieexplore.ieee.org/document/7838537.

[37] P. Harvey-Collard, R. M. Jock, N. T. Jacobson, A. D. Baczewski, A. M. Mounce, M. J. Curry, D. R. Ward, J. M. Anderson, R. P. Manginell, J. R. Wendt, M. Rudolph, T. Pluym, M. P. Lilly, M. Pioro-Ladrière, and M. S. Carroll, All-electrical universal control of a double quantum dot qubit in silicon MOS, in 2017 IEEE International Electron Devices Meeting (IEDM) (IEEE, New York, 2017), pp. 36.5.1-36.5.4, https://ieeexplore.ieee.org/document/ 8268507.

[38] X. Hao, R. Ruskov, M. Xiao, C. Tahan, and H. Jiang, Electron spin resonance and spin-valley physics in a silicon double quantum dot, Nat. Commun. 5, 3860 (2014).

[39] S. Shevchenko, S. Ashhab, and F. Nori, Landau-ZenerStückelberg interferometry, Phys. Rep. 492, 1 (2010).

[40] S. Mehl and D. P. DiVincenzo, Inverted singlet-triplet qubit coded on a two-electron double quantum dot, Phys. Rev. B 90, 195424 (2014).

[41] See Supplemental Material at http://link.aps.org/ supplemental/10.1103/PhysRevLett.122.217702 for additional details about slow and rapid adiabatic passage, pulse sequence details, measurement of the $S-T_{\text {- gap, relaxation }}$ time, dynamic nuclear polarization, and spin-orbit interaction model, which includes Refs. [42-44].

[42] C. Zener, Non-adiabatic crossing of energy levels, Proc. R. Soc. A 137, 696 (1932).

[43] C. Fasth, A. Fuhrer, L. Samuelson, V. N. Golovach, and D. Loss, Direct Measurement of the Spin-Orbit Interaction in a Two-Electron InAs Nanowire Quantum Dot, Phys. Rev. Lett. 98, 266801 (2007).

[44] O. E. Dial, M. D. Shulman, S. P. Harvey, H. Bluhm, V. Umansky, and A. Yacoby, Charge Noise Spectroscopy Using Coherent Exchange Oscillations in a Singlet-Triplet Qubit, Phys. Rev. Lett. 110, 146804 (2013).

[45] Z. Qi, X. Wu, D. R. Ward, J. R. Prance, D. Kim, J. K. Gamble, R. T. Mohr, Z. Shi, D. E. Savage, M. G. Lagally, M. A. Eriksson, M. Friesen, S. N. Coppersmith, and M. G. Vavilov, Effects of charge noise on a pulse-gated singlet-triplet $S-T_{-}$qubit, Phys. Rev. B 96, 115305 (2017).

[46] N. E. Bonesteel, D. Stepanenko, and D. P. DiVincenzo, Anisotropic Spin Exchange in Pulsed Quantum Gates, Phys. Rev. Lett. 87, 207901 (2001).

[47] M. Milivojević, Symmetric spin-orbit interaction in triple quantum dot and minimisation of spin-orbit leakage in CNOT gate, J. Phys. Condens. Matter 30, 085302 (2018).

[48] J. Klinovaja, D. Stepanenko, B. I. Halperin, and D. Loss, Exchange-based CNOT gates for singlet-triplet qubits with spin-orbit interaction, Phys. Rev. B 86, 085423 (2012).

[49] T. Tanttu, B. Hensen, K. W. Chan, H. Yang, W. Huang, M. Fogarty, F. Hudson, K. Itoh, D. Culcer, A. Laucht, A. Morello, and A. Dzurak, Controlling spin-orbit interactions in silicon quantum dots using magnetic field direction, arXiv:1807.10415. 